\title{
DIGITALE MEDIEN UND LERNSZENARIEN IM DAF-UNTERRICHT ${ }^{1}$
}

ABSTRACT: In der neuesten Zeit kann man eine rasant wachsende Bedeutung des ELernens auf allen Bildungsebenen beobachten. Zahlreiche digitale Medien werden ohne Vorbereitung und Planung in kurzer Zeit erprobt und eingesetzt, und das Lernen durch Versuche und Irrtum gewinnt zunehmend die Oberhand. In diesem Aufsatz werden mittels der theoretischen Analyse (1) die Schlüsselbegriffe im Zusammenhang mit dem E-Lernen geklärt, (2) seine Vorteile und Nachteile, (3) seine Einsatzmöglichkeiten im DaF-Unterricht in Bezug auf die didaktischen Prinzipien (Kompetenzorientierung, Interaktionsorientierung, Förderung von autonomem Lernen, Handlungsorientierung, Lerneraktivierung, Lernerorientierung, interkulturelle Orientierung) angeführt, und (4) verschiedene digitale Lernszenarien mit dem Ziel vorgestellt, Lehrende bei einem bedachten und planvollen Einsatz der digitalen Medien im DaF-Unterricht zu unterstützen.

Schlüsselwörter: Blended Learning, E-Lernen, didaktische Prinzipien, Unterrichtsplanung.

\section{DIGITAL MEDIA AND LEARNING SCENARIOS IN TEACHING GERMAN AS A FOREIGN LANGUAGE}

ABSTRACT: E-learning has recently gained significant relevance at all levels of education. Various digital media have been tested and implemented without preparation and planning in a short span of time, and learning through trial and error has gained the upper hand. In this paper, the method of theoretical analysis shall be applied in order to (1) discuss the key concepts referring to e-learning, (2) name its advantages and disadvantages, (3) list possible ways of implementation regarding the didactic principles (competence, interaction, learner and action orientation, intercultural orientation, promotion of autonomous learning), and (4) introduce various digital learning scenarios. The aim of the paper is to offer support in deliberate and planned implementation of digital media in teaching.

Key words: Blended Learning, E-learning, didactic principles, lesson planning.

${ }^{1}$ Der Aufsatz ist im Rahmen des Republik-Projekts Nr. 01600 entstanden, das vom Ministerium für Bildung, Wissenschaft und technologische Entwicklung der Republik Serbien gefördert wird. 


\section{E-LERNEN UND DIGITALE MEDIEN}

E-Lernen oder E-Learning bezieht sich auf das elektronisch gestützte Lernen, wenn irgendeine Art von digitalem Material oder eine Verwendung von digitalen Kommunikationskanälen eingebracht wird (Grünewald 2010: 42). „Lernen“ ist ein recht umfangreicher und vielschichtiger Begriff, aber wenn von „dem absichtsvollen, planmäßigen und bewussten Lernen“ die Rede ist, wie wir es vor allem in Form von Unterricht kennen, meint man damit ,den bewussten Vorgang der Einprägung von Kenntnissen, der Aneignung und Entwicklung von Wissen, Erkenntnissen, Fertigkeiten, Verhaltensweisen und Haltungen" (Treml und Becker 2004: 106). E-Lernen ist demzufolge das Lernen, bei dem als technische Unterstützung zum Informationszugang digitale oder elektronische (Nadrljanski, Nadrljanski, Bilić 2009), neue (Bausch 2007) oder tertiäre Medien (Wermke 2006) verwendet werden. Dazu gehören vor allem Personal Computer (PC) mit CDs und DVDs sowie Mobilgeräte wie Notebook, Tablet oder Smartphone.

Digitale Medien können online oder offline genutzt werden bzw. mit oder ohne Internetzugang. $\mathrm{Zu}$ den Offlineangeboten gehören $\mathrm{CD}$ und DVD sowie verschiedene Softwares wie z. B. die Präsentationssoftware MS PowerPoint. Die Onlineangebote $^{2}$ umfassen z. B. Onlineübungen und -tests, Spielgeschichten, narrative Computerspiele oder Hyperfiction (Abraham und Kepser 2006: 173-175; Leubner, Saupe, Richter 2010: 202). Die Onlineangebote werden zudem in formale (Unterrichtsmaterialien, interaktive Onlineübungen, Onlinetests usw.) und informale (soziale Netzwerke, Chatrooms, Wiki, YouTube, Weblog, Onlinespiele usw.) differenziert (Meister und Shalaby 2014: 9-11). Bei der Onlinekommunikation unterscheidet man weiter zwischen der asynchronen (EMail, Foren, Wiki, Weblog) und synchronen (Chat, Video-Konferenz) Kommunikation. Video-Konferenzen können als Grundlage für Projekte der Telekollaboration und für das Lernen in Teletandems dienen (Müller-Hartmann 2007: 198-216). Teletandems sind für das Üben der Phonetik und Phonologie mit Muttersprachlern sehr gut geeignet, wie es Guglielmi und Blatešić (2011: 96) am Beispiel des Italienischen gezeigt haben. Lernende und Lehrende müssen nicht

\footnotetext{
2 Obwohl die Geschichte des Internets (vgl. Braun 2010) bereits im Jahr 1957 begonnen hat, erlebte es seinen weltweiten Durchbruch erst mit WWW im Jahr 1989. Für die privaten und kommerziellen Nutzer wurde das Internet ab 1991 freigegeben und Mitte der neunziger Jahre (Riechert 1998 und Braun 1998) wurde es auch schon im Deutschunterricht eingesetzt.
} 
mehr zur gleichen Zeit am gleichen Ort sein, was neue Möglichkeiten eröffnet und das Lernen grundsätzlich verwandelt.

Digitale Medien können als Instrument (didaktisch) und als (Lern)Gegenstand (erzieherisch) in den Lernprozess eingebunden werden. Im ersten Fall werden sie als Lernmaterialien bzw. Lernwerkzeuge verwendet, im zweiten sind sie Gegenstand einer Reflexion zu einem relevanten Thema wie z. B. Gefahren in sozialen Netzwerken, Fragen des Datenschutzes (Herzig 2014: 9), Einfluss der Medien auf das Lehren und Lernen usw. Im Folgenden werden sie ausschließlich als Lern- und Lehrinstrumente thematisiert.

\section{VORTEILE UND NACHTEILE DES E-LERNENS}

Als Vorteile des E-Lernens werden folgende angeführt: (1) Unabhängigkeit von Ort und Zeit (auf die Inhalte kann überall und jederzeit zugegriffen werden), (2) Flexibilität (die Inhalte sind leicht und schnell veränderbar), (3) Wiederverwertbarkeit (die Inhalte können in andere Lernumgebungen übernommen werden), (4) Distribuierbarkeit (die Inhalte können leicht verteilt werden), (5) Archivierbarkeit (die Inhalte können gespeichert und katalogisiert werden), (6) Interaktivität (die Teilnehmer können selbständig Parameter verändern und auf das Lerngeschehen Einfluss nehmen), (7) Hypertextualität (Informationen können vernetzt werden) (Grünewald 2010: 42).

Die räumliche Unabhängigkeit der Nutzung und die Möglichkeit der asynchronen Nutzung der digitalisierten Materialien werden als erhebliche Vorteile angesehen, was dem E-Lernen einen überzeugenden Mehrwert im Vergleich zum Präsenzunterricht sichert. Mit einer Vorlesung, die synchron im Hörsaal gehalten, zeitgleich ins Internet übertragen und später als Videocast angeschaut werden kann, werden den Lernenden mehrere räumliche und zeitliche Möglichkeiten der Aufnahme geboten. Die Mehrzahl der digitalisierten Lernelemente und Lernformate kann prinzipiell ortsunabhängig und asynchron eingesetzt werden und geht mit einem hohen Grad an Flexibilität für die Lernenden einher. In der gegenwärtigen Zeit der Globalisierung sind solche Vorteile, die die digitalen Medien bieten, von großer Bedeutung, weil sie den Lernenden ein mobiles Lernen bzw. eine größere Entscheidungsfreiheit, Selbständigkeit und Verantwortung in Bezug darauf ermöglichen, wann, wo und wie sie lernen können oder wollen (Wannemacher et al. 2016: 56-57). Das führt zur Minderung des Zeit- und Kostenaufwands (z. B. für Reisen) und zur Steigerung der Produktivität und Effizienz. Besonders für junge oder vielbeschäftigte Eltern, bei globalen Krisen 
oder persönlichen Schicksalsschlägen erweist sich das E-Lernen als eine Plattform, die den (generell oder temporär) Benachteiligten gleiche Bildungschancen bietet.

Für die Lehrenden, die viel Zeit und Energie für die Aufbereitung der Materialien brauchen, sind die Flexibilität, Wiederverwertbarkeit, Distribuierbarkeit und Archivierbarkeit der Inhalte von großer Bedeutung. Damit ist aber auch ein Nachteil verbunden: die kreierten Inhalte müssen digitalisiert und zugänglich gemacht werden, und nicht alle Lehrenden verfügen über entsprechende IT-Kompetenzen. Hierfür bestehen zwei Lösungsmöglichkeiten: entweder sich weiterzubilden, oder das Material im Team mit einem IT-Experten aufzubereiten.

Ein großer Vorteil des E-Lernens ist auch die Möglichkeit der hypertextuellen Verbindung. Das Hypertextkonzept ist schon seit den 1930er Jahren bekannt, Tim Berners-Lee hat es aber erst für das Internet mit dem Ziel angepasst, verschiedene Dokumente, in denen Wissen zu Experimenten, Technologien oder organisatorischen Aspekten festgehalten ist, miteinander zu verknüpfen und ein Informationssystem aufzubauen, welches das Sammeln, Verwalten und das einfache Aktualisieren von Wissen unterstützt (Braun 2010: 205). Bei diesem Konzept ist nicht nur das Vernetzen von Informationen zu berücksichtigen, sondern auch die Förderung des vernetzten und divergenten Denkens bei den Lernenden, ihrer Wissbegier und Neubegier sowie ihrer Forschungskompetenzen.

Nach didaktischen Kriterien ist der hohe Grad an Interaktion zweifellos der bedeutendste Vorteil des E-Lernens. Dabei ist eine Interaktion im doppelten Sinne gemeint: einerseits mit der technologischen Umgebung in der Rückkoppelung zwischen Mensch und Computer, andererseits mit der sozialen und räumlichen Umgebung. Die Interaktivität einer Lernumgebung kann verschiedene Steuerungsmöglichkeiten umfassen, von der Möglichkeit, die Repräsentationsform von Inhalten oder die Inhalte selbst zu verändern, bis zur Möglichkeit, Lernobjekte bzw. Inhalte selbst zu konstruieren. Der Austausch der Lernenden untereinander oder mit den Lehrenden in der sozialen und räumlichen Umgebung variiert in unterschiedlichen Lernformaten. Digitale Lernumgebungen umfassen interaktive Elemente wie Kommunikations-, Kollaborations-, Content Sharing- und Assessmenttools. Einen besonders hohen Grad des Austauschs ermöglichen Mehrbenutzer-Anwendungssysteme zur Kollaboration wie Google Docs und Kommunikationstools wie ein Videokonferenzdienst, soziale Medien, Chat, Diskussionsforum oder E-Mail. Der Austausch mit der räumlichen Umgebung steht bei Lernelementen und Lernformaten wie virtuellen Laboren oder Augmented Reality zweifelsohne im Vordergrund (Wannemacher et al. 2016: 53). 
Durch die Arbeit mit digitalen Videos werden sowohl Hörverständnis und Sprechkompetenz als auch grammatische, lexikalische und pragmatische Kompetenzen gefördert, doch die Vorteile der schriftlichen CMC (eng. computermediated communication oder computergestützte Kommunikation) für die Förderung der kommunikativen und interkulturellen Kompetenzen sind ebenfalls unbestreitbar. Eine Untersuchung mit 38 Englischlernern an der Universität Puerto Rico at Mayaguez (Sullivan and Pratt 1996: 491-501) hat beispielsweise gezeigt, dass in einer Computergruppe die Lerner die Diskussion mit 85\% der Beiträge dominierten, während in der mündlichen Gruppe nur 35\% der Turns von ihnen gestaltet wurde. Ein weiterer Vorteil der schriftlichen Modalität ist ein größeres $\mathrm{Ma} ß$ an Reflexivität und an Sicherheit, sodass Lerner kontrollierter und bewusster miteinander kommunizieren, sich stärker beteiligen, mehr Neues ausprobieren und damit ihre fremdsprachliche Kompetenz erweitern (Rösler und Tschirner 2005: 77).

Als Hauptnachteil des Einsatzes der digitalen Medien wird der Mangel an direktem Kontakt (Mimik, Gestik, Berührung, Bewegung) angesehen (Meister und Shalaby 2014: 11). Der berühmte Psychologe Albert Mehrabian hat jahrelang (1972-1981) Forschungen zur Kommunikation mit dem Ergebnis durchgeführt, dass 55\% der Nachricht durch nicht-verbale Kommunikation (Mimik und Gestik), 38\% durch Ton und Stimme und nur 7\% durch Inhalt und Worte vermittelt wird (Mehrabian 1971: 51). Demzufolge geht mit dem Einsatz der digitalen Medien der größte Teil der Nachricht an Lernende verloren.

In Bezug auf den Wissenserwerb und die Problemlösefähigkeiten bzw. Transferfähigkeit ist ein höherer Lernerfolg zu erwarten, wenn Informationen als Text-Bild-Kombination gestaltet und präsentiert werden, als wenn sie nur als Text dargeboten werden. In Bezug auf die Sinnesmodalitäten ist festzuhalten, dass ein höherer Lernerfolg $\mathrm{zu}$ erwarten ist, wenn Informationen simultan auditiv und visuell präsentiert werden, als wenn sie nur visuell (als geschriebener Text und als Bild bzw. als Animation) oder nur auditiv (als gesprochener Text) dargeboten werden (Herzig 2014: 12). Digitale Medien, die die Verbindung von Text, Bild und Ton ermöglichen (Video-Aufnahmen, Video-Konferenzen, Augmented Reality, computergestützte Simulationen usw.), werden demzufolge didaktisch sehr hoch bewertet, aber in Bezug auf den aktiv lehrenden Menschen bleibt die Maschine doch Maschine und dieser Nachteil lässt sich auch nicht beheben. 


\section{E-LERNEN IM DAF-UNTERRICHT}

Digitale Medien sind vor allem für ausländische Deutschlerner attraktiv und effektiv, weil dadurch sprachliche und kulturelle Phänomene leicht verständlich gemacht werden können, und sie eine Abwechslung gegenüber dem herkömmlichen Lernmaterial und Präsenzunterricht bieten. Obwohl digitale Medien der Kultur der jungen Lernenden angehören, fördern sie sowohl die Medienkompetenz der Lerner als auch der Lehrer (Son 2004: 76-77). Ein didaktisches Problem bei ihrem Einsatz ist, dass eine Vielzahl der Materialien im Prinzip keine wesentlichen Unterschiede zu den herkömmlichen Printmedien aufweist, obwohl von digitalen Materialien ein anregender und abwechslungsreicher Unterricht erwartet wird. Oft wirken sie in entgegengesetzter Richtung, sodass von einem „didaktischen Rückschritt“ gesprochen werden kann (Son 2004: 79).

Aus dem Grund sollte der Einsatz der digitalen Medien geplant werden. Die Auswahl der digitalen Medien und Angebote sollte nicht aufs Geratewohl oder per Zufall getroffen werden, sondern mit Hinblick auf die Lehr- und Lernziele und mit Berücksichtigung von didaktisch-methodischen Prinzipien der Kompetenzorientierung, Interaktionsorientierung, Förderung von autonomem Lernen, Handlungsorientierung, Lerneraktivierung und -orientierung und interkulturellen Orientierung (Ende et al. 2013: 14-115). Die üblichen Merkmale eines guten Unterrichts sind auch für den digitalen Unterricht maßgebend: (1) Planung der echten Lernzeit zum Erreichen des Lernziels (die Zeit für das Lernen, ohne Ablenkungen, Störungen oder Nebensächlichkeiten), (2) vorbereitete Lernumgebung, (3) klare Strukturierung, (4) lernförderliches Klima, (5) inhaltliche Klarheit, (6) sinnstiftendes Kommunizieren, (7) individuelles Fördern, (8) Methodenvielfalt, (9) transparente Leistungserwartung und (9) intelligentes Üben (Lernstrategien werden vermittelt, Übungen sprechen Lernende an und machen ihnen Spaß). Obwohl nicht alle in jeder Unterrichtsstunde gleich relevant sind, sollten sie bei der Unterrichtsplanung mitbedacht werden (Brash und Pfeil 2017: 22-23). Digital heißt nicht sofort erfolgreich, sodass Unterrichtsplanung weiterhin eine wichtige Rolle spielt, was viele Lehrende aus dem Blick verlieren.

Die acht Leitfragen der Unterrichtsplanung sind: (1) Was will ich erreichen? (2) Wo stehen meine Lernenden? (3) Was tun die Lernenden, um das Lernziel zu erreichen? (4) Wie arbeiten die Lernenden zusammen? (5) Womit arbeiten die Lernenden? (6) Wie präsentiere ich die Lehr- bzw. Lernmaterialien? (7) Was tue ich als Lehrerin / als Lehrer? (8) Wie evaluiere ich das Erreichen von 
Lernzielen? (Brash und Pfeil 2017: 80) Lehrende sollten im Hinblick auf diese Fragen eine Auswahl unter den zahlreichen digitalen Angeboten treffen.

Elemente des E-Lernens bewegen sich auf einer breiten Skala: von den Aufgaben mit dem Einsatz der digitalen Medien - beispielsweise wenn Lernende die Ergebnisse einer Internetrecherche in einem Blog präsentieren - über interaktive Onlineübungen bis zu Lernplattformen als der komplexesten Form des E-Learning (Meister und Shalaby 2014: 9-11). Die Angebote zu E-Learning steigen stetig, sie sind sehr vielfältig und reichhaltig und bieten Raum für Kreativität (der Lernenden und Lehrenden), falls sie in angemessener Weise und kompetent verwaltet werden.

Durch den Einsatz des E-Lernens im DaF-Unterricht lassen sich sprachliche, (inter)kulturelle und digitale Kompetenzen fördern. Digitale Medien bieten authentisches Material zum Lesen und Hören sowie vielfältige Übungsmöglichkeiten für das Schreiben und Sprechen in der Fremdsprache, möglicherweise auch in Zusammenarbeit mit Muttersprachlern. Dadurch wird ebenfalls das kulturelle Lernen ermöglicht, falls die Inhalte sorgfältig ausgewählt werden. Interkulturelle Kompetenzen lassen sich durch den virtuellen Austausch (Virtual Exchange) fördern, wie es zurzeit im Rahmen des Erasmus+ Projekts angeboten wird (Erasmus+ Virtual Exchange 2020). Mithilfe des Projekts wird Lernenden ermöglicht, mit jungen Leuten weltweit von Zuhause aus zu kommunizieren.

Zahlreiche Studien zeigen, dass die positiven Wirkungen des Medieneinsatzes auch in überfachlichen Kompetenzbereichen Niederschlag finden: stärkere Kooperation, höhere Medienkompetenz, bessere Selbststeuerung und höhere kognitive Komplexität. Motivationale Effekte können ebenfalls gesteigert werden, sie sind aber zeitlich begrenzt, wenn sie sich bloß auf den Umgang mit den technischen Geräten und die begleitende Medienkompetenz beziehen (Herzig 2014: 13).

Interaktionsorientierung, Förderung von autonomem Lernen, Handlungsorientierung, Lerneraktivierung und -orientierung werden durch den Einsatz von digitalen Medien im Unterricht nicht immer erreicht. Digitale Medien werden zumeist als Datenträger benutzt und ihr hohes Potenzial wird leider nicht völlig ausgeschöpft. Zunächst muss der erste Schritt, die Bekanntmachung mit Web Tools und ihren Anwendungsmöglichkeiten, bewältigt werden, bevor sie didaktisch-methodisch angemessen in den Unterrichtsprozess eingebunden werden können.

Digitale Medien werden im Unterricht meist zum Zweck der Präsentation und Kommunikation eingesetzt. Diese Anwendung ist die einfachste und zurzeit 
die verbreitetste, wobei das keine Gewähr für einen interaktiven, lernerzentrierten Unterricht leistet. PowerPoint Präsentationen sind z. B. oft nur ein (relativ) modernes Mittel im Frontalunterricht, das der Veranschaulichung des Lerninhalts dient, nicht aber das Engagement der Lernenden erhöht. Zu dem Zweck können Präsentationen mit digitalen Medien, Übungen und Aufgaben kombiniert werden, wie es verschiedene Web Tools wie Nearpod oder Versal ermöglichen.

Das Angebot der interaktiven Lernmaterialien, die online zugänglich sind, reicht von digitalen Lehrbüchern, wie sie z. B. der Klett Verlag hergestellt hat, bis zu Übungen und Tests, die eine gute Möglichkeit zum selbständigen Lernen anbieten. In der Interaktion von Lernenden mit den Onlinelernmaterialien werden Lehrende in den Hintergrund gerückt und der Unterrichtsprozess wird zwangsläufig zum lernerzentrierten Lernprozess. Interaktive Übungen und Tests für Deutsch sind auf den Internet-Seiten der Verlage (z. B. Hueber Verlag, Schubert Verlag), Institute (z. B. Goethe-Institut, Österreich Institut) und Portale (z. B. Alumniportal Deutschland, derdieDaF Portal) zugänglich. Im derdieDaF Portal sind zusätzlich zahlreiche Apps und Tools für den DaF-/DaZ-Unterricht verlinkt, sodass Lehrende eigene Übungen und Aufgaben erstellen, und Lernende sie lösen können. Bei der Ausarbeitung von Aufgaben, Übungen, Quiz und Tests sollten Lehrende so viel wie möglich Blooms Lernzieltaxonomie berücksichtigen, damit die digitalen Medien nicht nur der reinen Wissensüberprüfung dienen, sondern Anlass zum aktiven und autonomen Lernen bieten.

Die Mehrheit der Tools sind per Smartphone zugänglich, sodass Smartphones immer mehr Anwendung im Unterrichtsprozess finden, wodurch der Bezug zu ihrer Lebenswirklichkeit hergestellt wird. Smartphones eignen sich besonders gut für die Gruppenarbeit, wobei in einer Gruppe mindestens ein Gerät gebraucht wird, um die Apps für das Lösen von Aufgaben, den Meinungsaustausch, das Sammeln von Ideen, das Erzählen von Geschichten, das Erstellen von Foto- und Video-Collagen, die Arbeit mit Comics usw. benutzen zu können (Anand und Mitra 2017: 19-24). Da nicht in jedem Klassenraum die entsprechende Zahl von PCs vorzufinden ist, müssen digitale Medien wegen der mangelnden technischen Ausstattung nicht unbedingt aus dem Unterrichtsprozess ausgeschlossen werden.

Zum kooperativen Lernen im DaF-Unterricht gibt es zahlreiche Tools wie Google Docs und Zoho (zum kollaborativen Schreiben) oder Perusall (zum gemeinsamen Lesen). Der Einsatz dieser Tools ermöglicht die Aufgaben- und Handlungsorientierung im Unterricht, sowie die Förderung der Kreativität und der Sprachkompetenzen. Video-Konferenzen und Videoaufnahmen bieten eine gute 
Möglichkeit zum Üben der Sprechkompetenz in der Fremdsprache (Aussprache, Redefluss usw.).

Lerninhalte und Lernmaterialien können auf einer Blog-Seite zusammengestellt und präsentiert werden. Diesbezüglich entstehen heutzutage zahlreiche Blogs zum Deutschlernen und zu anderen verwandten Themen und Konzepten. Bekannte und zum Teil kostenlose Blog-Seiten sind WordPress, Wix, Weebly, Blogger, tumblr und viele andere. Einen guten Überblick über die neun besten Blog-Seiten gibt Adelina Tuca (2019), wobei sie WordPress auf Platz eins setzt, die Seite, die sie selbst benutzt. Neustein und López (2019) benutzen ebenfalls diese Seite für ihren deutschlernerblog für Deutschlerner in aller Welt, der ein gutes Beispiel für den Einsatz des Blogs im Fremdsprachenlernen ist. Auf dem Blog gibt es zahlreiche Angebote zu „Hörverstehen, Leseverstehen, Wortschatz, Grammatik, Übungen, Prüfungen, Schreiben, Quiz, Musik, Videos, Bilder“ (Neustein und López 2019). In einem Projekt können Lernende auch selbst Blog-Seiten erstellen. Die Zahl der Blog-Seiten und der diesbezüglichen Möglichkeiten zum Lernen steigt zunehmend, wodurch sich die Seiten ihrem Konzept nach immer mehr den Lernplattformen annähern.

Lernplattformen (eng. Learning management system, LMS) dienen zur Aufnahme und Verarbeitung von Informationen und eignen sich für kommunikative, kooperative und kollaborative Aufgaben, die die Interaktion zwischen Lernenden und Lerninhalten, Lernenden untereinander und Lernenden und Lehrkräften fördern (Meister und Shalaby 2014: 9-11). Sie verbinden alle bisher genannten Möglichkeiten und Formen der digitalen Medien: Präsentationen, Kommunikation (Nachrichten, Forum, Chat), Übungen und Tests. Sie sind im Unterricht bereits sehr stark vertreten, wobei unter ihnen Moodle die bekannteste und die verbreitetste Plattform ist. Moodle weist die größte Zahl der Nutzer weltweit auf, nicht nur wegen der hohen Qualität, sondern auch wegen der kostenlosen Software, die institutionsgebunden genutzt werden kann. Neben Moodle sind weitere bekannte LMS: Blackboard, Canvas, Ilias und Google Classroom, ein ebenfalls kostenloses Angebot von Google, dessen Nutzung sehr einfach und praktisch ist.

An digitalem Medienangebot mangelt es nicht. Das Internet bietet eine Menge an Möglichkeiten, die Zahl der Tools steigt rapide und im heftigen Konkurrenzkampf verbessern die Hersteller ihre Softwares und bieten sie den Nutzern kostenlos oder zu günstigen Preisen an. Problematisch ist aber, dass die Auswahl und Anwendung dieser Angebote meistens intuitiv und planlos verlaufen, was dazu beigetragen hat, dass ihr Einsatz nicht immer sinnvoll ist und grundsätzlich keinen Beitrag zur Qualitätssicherung im Unterricht leistet. 
Obwohl die lerntheoretische Diskussion um den Stellenwert und das Innovationspotenzial der digitalen Medien von konstruktivistischen Theorieansätzen bestimmt wird, besteht eine Diskrepanz zwischen der theoretischen Konzeption und dem Alltag der Sprachlernsoftwares, weil die Hersteller von Sprachlernprogrammen in der Praxis traditionelle Wissensvermittlung und herkömmliche Drill-and-Practice-Übungen mit Programmmerkmalen kombinieren, die ein selbständiges, exploratives Lernen unterstützen. Bei vielen praktischen Anwendungen der digitalen Medien erkennt man einen Widerspruch zu den theoretischen konstruktivistischen Forderungen. Während der Herstellung der Softwares bestehen wenige oder keine Bezüge zu den Lerntheorien, Spracherwerbstheorien, didaktischen Theorien oder linguistischen Modellen (Rösler und Tschirner 2005: 75). Aus dem Grund wird in der Fachliteratur den digitalen Lernszenarien immer mehr Aufmerksamkeit geschenkt.

\section{LERNSZENARIEN IM DAF-UNTERRICHT}

\subsection{Szenario}

Der Begriff „Szenario“ wird vom lateinischen Wort „scaena“ (Bühne) bzw. „scaenarius“ (zur Bühne gehörig) abgeleitet und bezog sich ursprünglich auf die Szenenbeschreibung für ein Theaterstück, heute auch für Film und Oper. Ein Szenario besteht aus Anweisungen zum Ablauf der Handlung und zu dazugehörenden Orten, Ausstattung, Schauspielern, Requisiten (Baumgartner 2006: 238-239). Obwohl das Konzept der Inszenierung (im engeren Sinne des Wortes) aus dem Theaterbereich kommt, erfreut es sich heutzutage großer Beliebtheit im allgemeinen Sprachgebrauch und im öffentlichen Leben (Politik, Medien, Kunst und Kultur) (Küppers und Schmidt 2010: 116), sowie im Bereich der Bildung.

In der politischen Bildung bezeichnet das Szenario eine Methode (Bloemen, Lübbers, Porath 2010), ein vereinfachendes Konstrukt, das einen möglichen Weg in die Zukunft sowie einen möglichen zukünftigen Zustand beschreibt (Hradil 2012). Die Zukunftsorientierung ist von Anfang an diesem Konzept inhärent, da es, wie es Baumgartner (2006: 239) betont, einen geplanten Handlungsablauf schildert, Informationen und Anweisungen zur Aufführung gibt und damit einen präskriptiven (vorschreibenden) Charakter aufweist. Diese Komponente wird auch auf andere Bereiche übertragen. 


\subsection{Didaktisches Szenario}

Das In-Szene-Setzen wurde von der Allgemeinen Didaktik und Fremdsprachendidaktik nutzbar gemacht, besonders im Rahmen der reformpädagogischen Ansätze, die das ganzheitliche Lernen anstreben (Küppers und Schmidt 2010: 116). In diesem Kontext wurden die Konzepte des Didaktischen Szenarios, der Szenariendidaktik und des digitalen Lernszenarios ausgearbeitet, die sich zum Teil überlagern.

Das Konzept des Didaktischen Szenarios wird auf Grund der Analogie mit der Theater- und Filmwelt als „ein Skript für die Inszenierung eines bestimmten Lernarrangements“ (Baumgartner 2006: 239) definiert. Im Szenario werden nur die wesentlichen Merkmale herausgearbeitet und festgehalten, auch mit dem Ziel, die Vielzahl der (möglichen) unterschiedlichen didaktischen Situationen zu begrenzen. Analog zur Filmwelt, in der es eine Reihe von Gattungen (Genres) gibt, wird als ein weiteres Merkmal der Didaktischen Szenarien ihre inhaltliche Neutralität genannt, weil die Genres in der Entwurfsphase spezifischen dramaturgischen Gesetzmäßigkeiten unterliegen und einen übergreifenden Deutungsrahmen bei der aktuellen Durchführung abgeben. Die jeweiligen Genres sind inhaltsleer und enthalten nur typische Muster, die zu erwarten sind (Baumgartner 2006: 239). Demzufolge erfassen Didaktische Szenarien lediglich das Typische des Ablaufs und der Ausstattung und werden in unterschiedlichen Zusammenhängen mit verschiedenen fachlichen Inhalten angewendet (Baumgartner 2006: 240). Im Fremdsprachenunterricht wird dieses Konzept weiter differenziert und zur Szenariendidaktik ausgearbeitet.

\subsection{Szenariendidaktik}

Der Begriff der Szenariendidaktik ist mit dem Namen von Hans-Eberhard Piepho verbunden und zielt auf das Einfordern von schulisch inszenierten Lernkontexten hin, die zur Realisierung echter Kommunikationsabsichten führen (Küppers und Schmidt 2010: 116). Ursprünglich für den Englischunterricht entwickelt, hat die Szenariendidaktik ihre Eignung für den Unterricht in allen Fächern bewiesen, insbesondere für den Deutschunterricht mit heterogenen Lerngruppen. Mit dem grundlegenden Konzept der Lerneraktivierung ist sie zur Erarbeitung fachlicher Inhalte und der expliziten Förderung sprachlicher Ausdrucksfähigkeit auf allen Kompetenzstufen und für alle Altersgruppen geeignet. Im Rahmen eines Lernszenarios werden Lernenden (1) zu einem vorgegebenen Kernthema (2) unterschiedliche Aufgaben angeboten, (3) die sie im 
Team besprechen, die Arbeit und die Präsentation planen, (4) nach der Vorstellung des Arbeitsvorhabens (5) optimieren, (6) abschließend präsentieren und (7) reflektieren. Die Lernszenarien sind besonders gut für interkulturelles Lernen geeignet (Hölscher, Roche, Simic 2009: 48-49) und ihre primären Merkmale sind Offenheit und Flexibilität, da Szenarien keine festen Lerninhalte und vorbestimmten sprachlichen Lernziele haben. Das Anspruchsniveau ergibt sich aus den vorhandenen Vorkenntnissen der Lernenden, und die spezifischen Lernziele und Lerninhalte sollen zusammen mit ihnen bestimmt werden, nachdem sie sich mit dem Handlungsablauf bekannt gemacht haben und einschätzen können, was sie zur Bewältigung der Situation und zum Erreichen des Handlungsziels benötigen. Diese starke Lernerorientierung bedeutet, dass die spezifischen Lerninhalte im Prinzip jedes Mal neu bestimmt werden und die strukturierende Rolle eines Lehrmittels in der Unterrichtsplanung wegfällt. Ebenso gibt es keine „passenden“ vorproduzierten Lernunterlagen zu den einzelnen Szenarien - z. B. in der Form von Arbeitsblättern (Hagenow-Caprez 2013: 47).

Das Potenzial der Inszenierung im Fremdsprachenunterricht liegt im Aufbrechen der ritualisierten Unterrichtssprache, in der Vermittlung alltagstauglicher Sprechhandlungskompetenz zum situationsgerechten, interkulturell-angemessenen und spontanen Sprechen und in der Aktivierung individueller und ganzheitlicher Kommunikationspotenziale. Bei der Realisierung der inszenierten Lernkontexte setzt man eine reflexive Einbettung des ganzen Lernprozesses voraus. Das Lernen als Erkenntnis, Einsicht oder Veränderung eines Standpunktes soll sich nicht lediglich in der abschließenden Feedback- oder Reflexionsphase manifestieren. Die Inszenierung soll sich an der ganzheitlichen, verbalen und nonverbalen Kommunikation orientieren und die Lernenden als Gruppe aktivieren (Küppers und Schmidt 2010: 117).

Neue Potenziale für die Inszenierungsprozesse in Lehr- und Lernkontexten einer Fremdsprache ergeben sich durch die digitalen Medien und Internetanwendungen wie Audio- und Videoblogs, Podcasts, Videoportale und Social Network Sites. Dadurch eröffnen sich neuartige Formen des Aufbaus und der Pflege der Informations- und Beziehungsnetzwerke, der multimedialen, kreativen Selbst-Inszenierung von Individuen sowie der vielfältigen Präsentation schriftlicher wie mündlicher Inhalte, die eigenständig von Lernenden für ein real existierendes Publikum produziert werden. Das Internet erweist sich als ein fremdsprachlicher Aktionsraum und die Lernenden inszenieren dabei (multimedial) sich selbst, ihre kulturelle Identität, ihre Ideen und Einstellungen für eine im Vergleich zum geschützten Raum des Klassenzimmers weitaus größere 
Öffentlichkeit (Küppers und Schmidt 2010: 116-117). Damit eröffnet sich ein neues Feld, in das ein neuer Begriff eingeführt wird: (digitale) Lernszenarien.

\subsection{Lernszenarien}

Brash und Pfeil (2017: 12-15) unterscheiden drei Lernszenarien (Formen des digitalen Lernens), je nachdem ob in der Freizeit oder im beruflichen Kontext, zufällig oder mit einer konkreten Frage gelernt wird. Beim ungesteuerten Lernen spielt sich der Lernprozess außerhalb eines Unterrichtskontextes und ohne die Unterstützung einer Lehrperson $a b$. Wenn dabei der Wissenserwerb aufgrund eines spezifischen Interesses oder einer zu füllenden Wissenslücke verläuft, nennt man diese Form informelles Lernen. Im Zusammenhang damit steht auch das spielbasierte Lernen oder Game-based Learning, bei dem Inhalte so aufbereitet werden, dass man sie spielerisch lernen kann. Jegliches Lernen mithilfe von Mobilgeräten (Smartphone, Tablet) wird mobiles Lernen genannt (Brash und Pfeil 2017: 13).

Lernprozesse, die in einem institutionalisierten Rahmen mit Unterstützung der Lehrperson stattfinden und mit einem definierten Lernziel verbunden sind, nennt man gesteuertes Lernen (Präsenzunterricht, Blended Learning-Format oder komplett online). Von hybridem Lernen wird gesprochen, wenn im Unterricht der Zugang zum Internet möglich ist und Informationen und Materialien gezeigt werden, oder wenn das gemeinsame Lernen über den physischen Unterrichtsraum hinaus auf einen Blog oder eine Lernplattform erweitert wird. Digitale Medien werden dabei didaktisch-methodisch sinnvoll in den Unterricht integriert, aber hybrides Lernen an sich legt noch nicht das Kursformat fest. Ein Format im Bereich des hybriden Lernens ist das Blended Learning, in dem der Unterricht klar in Präsenz- und Onlinephasen unterteilt wird, die sinnvoll aufeinander folgen und inhaltlich miteinander verbunden sind. Onlinekurse können sowohl asynchrone als auch synchrone Phasen beinhalten, wie z. B. in einem virtuellen Lernraum (VideoChat und virtuelle Präsenzsitzung) (Brash und Pfeil 2017: 14).

Die Lehrkraft ist, wie bisher, weiterhin präsent und wichtig, aber sie tritt stärker in den Hintergrund, während der Lernprozess und die Lerninhalte nun im Zentrum stehen und Lernende mit geeigneten Mitteln und Methoden unterstützt werden, neues Wissen zu erwerben oder vorhandenes Wissen auszubauen (Brash und Pfeil 2017: 14-15). Die Angst, dass die Lehrenden im digitalen Unterricht nicht mehr gebraucht werden, ist unbegründet, wenn sie Schritt mit den neuen Tendenzen halten und sich dem lebenslangen Lernen verpflichten bzw. sich anpassen und flexibel bleiben. Für die Lehrenden, die beim traditionellen 
Unterricht und bei traditionellen Medien bleiben möchten, ist diese Befürchtung zweifelsohne begründet.

Arnold et al. (2018: 137-138) verwenden den Begriff der Lernszenarien für die Beschreibung pädagogischer Verhältnisse bzw. für die Beziehungen zwischen den beteiligten Personen und der Verantwortlichkeiten für den Lernprozess. Dabei unterscheiden sie drei Tendenzen: (1) Dominanz der Lehrenden, (2) Dominanz des Lernens gegenüber dem Lehren und (3) Dominanz des Kommunikativen gegenüber dem Individuellen. Als Orientierung für die Gestaltung von Lernszenarien werden Lernaufgaben eingesetzt.

\subsection{Digitale Lernszenarien}

Dank der digitalen Medien wurden verschiedene Lernelemente und formate entwickelt wie z. B. adaptives Lernen, Augmented Reality, E-Lecture (Office- oder Studio-Setting), E-Portfolio, freie Lernmaterialien (Open Educational Resources), Game-based Learning, Inverted Classroom, mobiles Lernen, Nutzung sozialer Medien, Online-Peer- und kollaboratives Lernen, Onlineseminar, Onlinestudiengang, Open Course und MOOC, simulationsgestütztes Lernen, Virtual Reality und Vorlesungsaufzeichnung (Live-Digitized-Lecture). $\mathrm{Zu}$ den Merkmalen dieser Lernelemente und -formate zählen die Lern- und Lehrziele, Zielgruppe, Lernumgebung, curriculare Einbettung, und zu ihren Dimensionen die Lehrenden- / Lernendenrolle, der Grad der Interaktion, Virtualität und Medialität, die räumliche und zeitliche Flexibilität, Individualisierung, Granularität, Kostenund Arbeitsaufwand. Diese Merkmale und Dimensionen werden zu einem Strukturmodell verdichtet und aus ihrer Kombination werden auf einer höheren Aggregationsebene Lernszenarien gebildet (Wannemacher et al. 2016: 53-59). Trotz den zahlreichen Variationen unterscheidet man grundsätzlich drei Hauptszenarien: Präsenzunterricht, Fernunterricht und Blended Learning.

Beim Einsatz der digitalen Medien können Lernende und Lehrende zur gleichen Zeit am gleichen Ort (Präsenzunterricht) oder räumlich und zeitlich getrennt (Fernunterricht) sein. Fernunterricht (distance learning) wurde im deutschen Fernunterrichtschutzgesetz (Gesetz zum Schutz der Teilnehmer am Fernunterricht) aus dem Jahr 1977 folgendermaßen definiert: „Vermittlung von Kenntnissen und Fähigkeiten, wobei Lehrende und Lernende ausschließlich oder überwiegend räumlich getrennt sind, und der Lernerfolg durch den Lehrenden überwacht wird.“ (Art. 1) Zur Überbrückung der räumlichen Distanz zwischen den Lernenden und Lehrenden werden Medien eingesetzt: Druckerzeugnisse („Lehr oder Studienbriefe") mit Fragen und Aufgabenstellungen, die bearbeitet und zur 
Kontrolle zurückgeschickt werden („Einsendeaufgaben“) (Juchem-Grundmann 2010: 59-60). Im Hinblick auf die Flexibilität der Lernenden, die Arten der Interaktionen von Lernenden, Material und Tutor sowie die Synchronität und Asynchronität der Interaktion unterscheidet man vier Generationen des Fernlernens: (1) Printmedien, (2) Hörtexte, Video, Fernsehen, Radio und computerunterstütztes Lernen, (3) E-Mail, Audio- und Videokonferenzen sowie computervermittelte Kommunikation, (4) interaktive Multimedia- und Computersimulationen (Rösler und Tschirner 2005: 76). Wenn in regelmäßigen Abständen zusätzliche Präsenztermine zur Vertiefung der Inhalte, Klärung der Fragen und Förderung der sozialen Interaktion stattfinden, entsteht eine Mischform aus Fernunterricht und Präsenzunterricht. Der Großteil der heute unter Fernunterricht subsumierten Angebote entspricht diesem Konzept (JuchemGrundmann 2010: 59-60). Wenn der traditionelle Präsenzunterricht mit dem modernen E-Learning kombiniert wird, sodass die Vorteile beider verbunden werden, spricht man vom Blended Learning oder hybridem Lernen (Meister und Shalaby 2014: 9-11). Die Entwicklung erfolgreicher Blended Learning-Konzepte beruht auf einer ausgewogenen Mischung zwischen Präsenzunterricht, autonomen Phasen (u. a. mithilfe multimedialer Lernumgebungen) und Tandem- und ETandem-Angeboten, wobei eine wesentliche Rolle die Lernberatung einnehmen muss (Rösler und Tschirner 2005: 78). Diese Ausgewogenheit wird aber nicht immer erreicht, weil die lehrzentrierten Unterrichtsformen immer noch überwiegen.

Im „Anreicherung“-Szenario werden Lehrveranstaltungen mit digitalen Komponenten bloß bereichert, ohne dass die Präsenzlehre grundsätzlich verändert wird. Bei der „Integration“ handelt es sich um konventionelle Blended LearningAnsätze, bei denen sich die Präsenzphasen und digitalisierten Lernphasen gegenseitig ergänzen. In diesem Szenario wird den Lernenden mehr räumliche und zeitliche Flexibilität erlaubt, es werden Online-Communitys gebildet oder verschiedene Dokumente mittels einer Kollaborationssoftware gemeinsam bearbeitet. Im Szenario „Online-Lernen“ werden Lernangebote fast ausschließlich online bereitgestellt, mit einer kurzen oder keiner Präsenzphase. Die ersten drei Lernszenarien schließen sich gegenseitig aus, während die folgenden Szenarien mit anderen kombiniert werden können. Im Szenario „Interaktion und Kollaboration“ werden soziale Medien und Netzwerke sowie interaktive und kollaborative Anwendungen von verschiedenen Onlinediensten vorgesehen. Beim Szenario "Offene Bildungspraxis“ stehen freie Studienangebote zur Verfügung und die Lernenden bestimmen selbst, welche freien Lernmaterialien zur Verbesserung ihrer Lernerfahrungen beitragen sollten. Das Szenario „Spiel und Simulation“ umfasst 
alle Varianten des auf digitalisierten Spielen basierenden Lernens sowie der „digitalisierten Wirklichkeit“ (z. B. Virtual Reality). Im Szenario „Personalisierung“ wird eine Anpassung der digitalisierten Lernformate und Lernplattformen an individuelle Lernbedarfe ermöglicht. Zuletzt, das Szenario „Selbststudium“ umfasst alle Formen der digitalen Unterstützung von Prozessen des Selbststudiums (Wannemacher et al. 2016: 8-9) und entspricht völlig dem Prinzip des autonomen Lernens, der Handlung-, Aufgaben- und Lernerzentrierung.

$\mathrm{Zu}$ den aktuellen Ausprägungen bzw. Formen neuer Lernszenarien, in denen digitale Medien eingesetzt werden, gehören: (1) MOOCs (Massive Open Online Courses), die große Teilnehmerzahlen von mehreren Hundert bis zu Hunderttausenden Personen und offene Teilnahmeregelungen ohne Eingangsvoraussetzungen und meistens ohne Kursgebühren erlauben und im virtuellen Kursformat angeboten werden; (2) Inverted Classroom (umgedrehter Unterricht) oder Flipped Classroom ist so konzipiert, dass Lernende sich zu Hause selbständig und im eigenen Tempo die von Lehrenden digital zur Verfügung gestellten Inhalte aneignen, und im Unterricht diese durch (kollaborative) Übungen vertiefen; (3) das bereits erwähnte Game Based Learning (spielbasiertes Lernen) durch Computer- und Videospiele (Arnold et al. 2018: 147-151).

Je nachdem wer die Autorensoftware bedient, unterscheidet man drei Lernszenarien: (1) Beim tutoriellen Einsatz erstellt die Lehrperson interaktive motivierende Unterrichtseinheiten für Lernende, ohne viel Aufwand für die Korrektur und ohne richtige Betreueung. (2) Wenn jedoch die aktiv-erforschende Komponente durch die Aufwertung von Aktivitäten, die das entdeckende Lernen fördern, vorhanden ist, wird ein exploratives Lernszenario verwirklicht. (3) Wenn Autorenprogramme als dynamische Lernwerkzeuge eingesetzt werden, wird ein Lernszenario realisiert, das in hohem Maße zur Erfüllung konstruktivistischer Lernprinzipien beiträgt. In diesem Lernszenario avancieren die Lernenden zu Materialproduzenten, indem sie - im Kontext von Lernprojekten - digitale Lerndossiers im Sinne persönlicher „Lernmappen“ (E-Portfolios) mit Inhalten und Übungen zu verschiedenen Themenbereichen aufbereiten (Chrissou 2010: 62-63). Diese drei Lernszenarien bewegen sich vom lehrerzentrierten bis zum lernerzentrierten Konzept, bei dem erst das autonome Lernen gefördert wird.

In einer anderen Klassifikation wird zwischen Micro-Learning-Einheiten, Blended Learning und Communities of Practice unterschieden. Die MicroLearning-Einheiten sind didaktisch aufbereitete Themen, die als autonome Bausteine eines Qualifizierungsprozesses gehandhabt werden. Das Lernformat ist überwiegend als eine selbständige, kurze und unteilbare Lernsequenz konzipiert. Ein zeitlich umfangreicheres Format wird dann herausgearbeitet, wenn die 
Hintergründe des jeweiligen Themas dargelegt und der Kontext verstehbar gemacht werden soll. Dieses Format gilt als eine Form des technologiegestützten Lernens und ist in die Struktur eines sogenannten Web Based Training (WBT) einzuordnen (Rustemeier-Holtwick, Wolpert, Sieger 2015: 78). Blended Learning kombiniert die sozialen Aspekte des gemeinsamen Lernens aus den Präsenzphasen und die Effektivität und Flexibilität von elektronischen Lernformen des ELearning. Ein didaktisches Konzept bietet den Rahmen für die methodische und mediale Aufbereitung. Neben den Präsenzveranstaltungen werden die Lernprozesse digital über eine Lernplattform gesteuert und begleitet. Die Lernplattform ist die Basis und zentrale Ausgangsstation für unterschiedliche Lernangelegenheiten, wie z. B. für das Dokumentieren des eigenen Lernprozesses und der gruppenbezogenen Arbeits- und Rechercheergebnisse (Rustemeier-Holtwick et al. 2015: 86). Communities of Practice werden als über einen langen Zeitraum bestehende Personengruppen verstanden, die Interesse an einem gemeinsamen Thema haben und Wissen gemeinsam aufbauen und austauschen wollen. Die Teilnahme ist freiwillig und das gemeinsame Thema wird als das Verbindende oder das Gemeinschaftliche von den Mitgliedern der Community selbst hervorgebracht. Communities of Practice entwickeln sich nicht aufgrund formaler Zugehörigkeit, sondern aus unterschiedlichen Interessens- und Bedürfnislagen (RustemeierHoltwick und Wolpert 2015: 110). Deshalb sind sie nicht für den Unterrichtsprozess relevant, sondern sie eignen sich eher für außercurriculare Aktivitäten. Diese Klassifikation umfasst (1) die Lernformate, bei denen digitale Medien bloß als Bereicherung des Frontalunterrichts verwendet werden, (2) die Lernformate, in denen es zur produktiven Integration der digitalen Medien kommt und (3) das Selbststudium, das sich außerhalb des formalen Unterrichts abspielt.

Trotz verschiedener Bezeichnungen handelt sich grundsätzlich um drei Lernszenarien, die sich von einer digitalisierten Variante des lehrerzentrierten Unterrichts über einen digitalen lernzentrierten Unterricht bis zu einer Kombination der beiden Unterrichtstypen erstrecken. Die Digitalisierung impliziert eine Modernisierung bezüglich der Medien, leistet aber keine Gewähr für eine konzeptionelle Innovation. Inwieweit sie realisiert wird, hängt davon $a b$, zu welchem Integrationstyp Lehrende gehören.

In Bezug auf die Veränderung der bisherigen Unterrichtsgestaltung unterscheidet man fünf Integrationstypen von Lehrenden, die mehr oder weniger große Veränderungen in ihren didaktischen Handlungsmustern beim Einsatz digitaler Medien vornehmen. Lehrende des ersten Typs (Subsumption unter lehrerzentrierten Unterricht) sehen sich in der Rolle der Wissensvermittler und strukturieren bzw. kontrollieren den Unterrichtsverlauf stark. Digitale Medien 
haben eher die Funktion des Arbeitsheftes und werden nur phasenweise ähnlich wie klassische Medien eingesetzt. Der zweite Lehrertyp (Fokus auf Medienkompetenz und Technik) schreibt dem Erwerb von Medienkompetenz eine hohe Bedeutung zu und erlebt eine Veränderung des Unterrichts an den Stellen, wo es insbesondere um die Vermittlung von technischen Kenntnissen und Fähigkeiten geht. Obwohl die sinnvolle Nutzung des Laptops zur Vermittlung fachlicher Inhalte für sie problematisch ist, sind sie zu Veränderungen des Unterrichts bereit und grundsätzlich aufgeschlossen. Beim dritten Typ (Curricular-inhaltlicher Fokus) stehen curriculare Inhalte im Fokus. Diese Lehrenden versuchen die Potenziale des Computers zur Erarbeitung bestimmter Inhalte im Unterricht sinnvoll zu nutzen. Die Lehrenden des vierten Typs (Didaktisch-methodischer Fokus) achten auf die Verbindung von Medium, Methode und Inhalt im Unterricht, reflektieren über die Veränderung der Inhalte und Methoden zwecks der Verbesserung von Lernprozessen bzw. des Unterrichts, auch wenn sie dabei von curricularen Inhalten abweichen. Lehrpersonen des fünften Typs (konstruktivistische Integration) sehen Inhalts-, Methoden- und Medienentscheidungen als ein ganzheitliches Wirkungsgefüge und sind an der qualitativen Verbesserung von Unterricht interessiert. Sie haben bereits zuvor lernerzentrierte und stark konstruktivistische Unterrichtsmethoden eingesetzt, die nun durch Laptops zusätzlich vereinfacht und qualitativ verbessert werden. Die Lehrer des fünften Typs zeigen sich innovativ und haben hohe Selbstwirksamkeitserwartungen an den erfolgreichen Einsatz von Computern im Unterricht (Schaumburg 2003: 169-178; Herzig 2014: 14-15).

Obwohl Lehrende nicht mehr im Zentrum stehen, bleiben sie die Leiter des ganzen Lernprozesses, die ihre Kontrolle aufgeben, Richtlinien geben und eine Beratungsfunktion übernehmen d. h. zu Moderatoren und Kompetenzvermittlern werden. Im DaF-Studium ist das höchst sinnvoll, da die Lehrenden zwischen dem authentischen fremdsprachlichen Material und den Lernenden vermitteln können, die Phasen des E-Lernens einplanen und den Lernenden freie Hand bei der Auswahl der online zugänglichen Inhalte überlassen können, damit sie sprachliche und (inter)kulturelle Kompetenzen im Selbststudium erwerben. Der Prozess der digitalen Modernisierung sollte daher mit der Aneignung des benötigten Wissens und der digitalen und didaktisch-methodischen Kompetenzen seitens der Lehrenden anfangen, begleitet von ihrer Selbstreflexion und der Anpassung des eigenen Lehrstils an die neuen Umstände auf dem Weg zur Innovation. 


\subsection{Digitales Lerndrehbuch}

Neben dem Begriff Lernszenario ist in der Fremdsprachendidaktik auch die teils synonyme Bezeichnung Lerndrehbuch geläufig. Erstens meint man damit eine Verlaufsübersicht, die Freiräume für Unvorhergesehenes enthält. Das Lerndrehbuch klärt, welche Arbeitsschritte wann und wo (im Präsenzstudium, im begleitenden Selbststudium oder im autonomen Selbststudium) vollzogen werden sollen, damit der Lernprozess kontinuierlich, zielorientiert und inhaltlich folgerichtig verläuft. Im idealen Fall informiert es über den Zeitpunkt und die Inhalte formativer und summativer Lernnachweise, über allfällige Integrationstage bei fächerübergreifenden Modulen und über weiterführende Quellen. Die Form eines Lerndrehbuchs soll dem Stil der Lehrenden und dem Inhalt entsprechen (Pfäffli Tanner 2015: 135). In dieser Bedeutung ist der Begriff zu vermeiden, da er in einem verwandten Kontext benutzt wird und zur terminologischen Verwirrung führen kann.

Zweitens dient das Drehbuch für E-Learning analog zum Konzept im Film als Grundlage für die gesamte Produktion einer E-Learning-Anwendung und bestimmt die Richtlinien für Softwareentwickler, Grafiker, Animatoren und Screendesigner. In dem Drehbuch sollte abgebildet werden, was wann und wo passiert, wie die Sprechertexte lauten und welche Bildschirmseiten aufeinander folgen (Stocker 2013: 1), mit welchen Informationen, Aufgaben und Fragestellungen die Lernenden konfrontiert werden sollen und wie die Stofffülle eingegrenzt und logisch strukturiert werden kann. In einem Drehbuch sollten die Dramaturgie und Art der Inhalte zur Planung der Inhaltsrepräsentation innerhalb eines konkreten Lernmoduls festgehalten werden. In dem Kontext ist ein Drehbuch eine detaillierte Beschreibung der zu entwickelnden multimedialen Lerneinheit und dient dazu, die Inhalte zu modularisieren, wieder verwendbare Einheiten auszumachen sowie Visualisierungen, Verlinkungen und andere hypermediale Elemente zu planen. Das Drehbuch fungiert somit als Schnittstelle zwischen der inhaltlichen Konzeption und technischen bzw. grafischen Umsetzung, als eine Hilfeleistung für Autoren, um Ideen und Inhalte zu strukturieren und zu editieren (e-teaching.org 2015).

In einem Multimedia-Drehbuch werden für jede einzelne zu programmierende Bildschirmseite alle Inhalte, Elemente und Regieanweisungen festgehalten (Arnold et al. 2018: 175). Bei der Drehbuch-Erstellung unterscheidet man folgende Phasen: Erstellung eines Exposés, Erstellung eines Grobkonzepts und Erstellung eines Feinkonzepts, wobei die Konzeption und technische Realisierung von E-Learning-Inhalten nicht in einer Hand liegen (ebd.: 214-215). 
Hier wird die Unterstützung von Mediendesignern gebraucht, weil Lehrende meist über entsprechende Kompetenzen nicht verfügen. Sie bleiben aber die Produzenten des $\mathrm{zu}$ programmierenden Lernmaterials. Digitale Lernszenarien sind ausschließlich Produkte von Lehrenden und deshalb sind sie von digitalen Lerndrehbüchern klar abzugrenzen.

\section{SCHLUSSFOLGERUNG}

Das E-Lernen ist zweifellos ein fester Bestandteil des DaF-Unterrichts geworden. Der Einsatz der digitalen Medien im Unterricht hat viele Vorteile - von der Unabhängigkeit von Zeit und Raum über Flexibilität, Wiederverwertung, Distribution, Archivierung und hypertextuelle Vernetzung der Inhalte bis zur Interaktivität. Der Mangel der nicht-verbalen und para-verbalen Aspekte lässt sich zum Teil durch Video-Aufnahmen und Video-Konferenzen nachholen. Die ideale Lösung wäre jedoch die Kombination des Präsenzunterrichts und des Fernunterrichts bzw. das Konzept des Blended Learning.

Durch digitale Medien können Lernende ihre Sprachkompetenzen, (inter)kulturellen Kompetenzen, Medienkompetenz und solche Schlüsselkompetenzen, wie es Teamarbeit und Selbststeuerung sind, fördern. Damit das Potenzial der digitalen Medien vollkommen ausgeschöpft wird, sollten digitale Medien nicht planlos verwendet werden, sondern im Sinne der Szenariendidaktik - mit dem Ziel, die Unterrichtsprache zu vermeiden und die Aufgaben den Interessen und dem Wissensniveau der Lerner anzupassen.

Der Einsatz von digitalen Medien soll nach einem digitalen Lernszenario verlaufen d. h. nicht bloß als Anreicherung des Präsenzunterrichts und nicht nur tutoriell dienen, weil solche Lehr- und Lernformate wenig vom traditionellen lehrzentrierten Unterricht abweichen. Lehrende sollten den Lernenden mittels der digitalen Medien eher eine Möglichkeit zum Selbststudium und zum konstruktiven Lernen bieten. Dabei ist es ausschlaggebend, dass Lehrende offen für neue Medien, ihre Vorteile und Nachteile sind, bei der Unterrichtsplanung die didaktischmethodischen Prinzipien berücksichtigen und verschiedene Szenarienkonzepte kennen, damit der Lernprozess sinnvoll und effizient durchläuft. In dem Bereich bleiben ihre fachlichen und didaktisch-methodischen Kompetenzen nicht nur weiterhin gefragt, sondern sogar erforderlich und unersetzlich. 


\section{LITERATUR}

Abraham, U. und Kepser, M. (2006). Literaturdidaktik Deutsch. Berlin: ESV.

Anand, M. und Mitra, A. (2017). Mobiltelefone im DaF-Unterricht mit grossen Schulklassen. Fremdsprache Deutsch 56: 19-25.

Arnold, P., Kilian, L., Thillosen, A., Zimmer, G. (2018). Handbuch E-Learning: Lehren und Lernen mit digitalen Medien. Bielefeld: W. Bertelsmann Verlag. Baumgartner, P. (2006). E-Learning-Szenarien: Vorarbeiten zu einer didaktischen Taxonomie, in E-Learning - alltagstaugliche Innovation?, hrsg. v. E. Seiler Schiedt, S. Kälin, Chr. Sengstag (Münster: Waxmann): 238-247.

Bausch, K-R., Christ, H., Krumm, H-J. (Hrsg.) (2007). Handbuch Fremdsprachenunterricht. Tübingen und Basel: A. Francke Verlag.

Bloemen, A., Lübbers, J., Porath, J. (2010). Szenario-Methode, in Methodenpool, hrsg. v. K. Reich. Zugriff am 23. 3. 2020. URL: <http://methodenpool.unikoeln.de >.

Brash, B. und Pfeil, A. (2017). Unterrichten mit digitalen Medien. München: Goethe-Institut.

Braun, A. (1998). Die Nutzung des Internet für den DaF-Unterricht. Info DaF 25/ 1: $72-84$.

Braun, T. (2010). Geschichte und Entwicklung des Internets. Spektrum 33/ 3: 201207.

Chrissou, M. (2010). Technologiegestützte Lernwerkzeuge im konstruktivistisch orientierten Fremdsprachenunterricht: Zum Lernpotenzial von Autoren- und Konkordanzsoftware. Hamburg: Verlag Dr. Kovač.

Ende, K., Grotjahn, R., Kleppin, K., Mohr, I. (2013). Curriculare Vorgaben und Unterrichtsplanung. München: Goethe-Institut und Klett-Langenscheidt.

Erasmus+ Virtual Exchange (2020). Zugriff am 3. 4. 2020. URL: $<$ https://europa.eu/youth/erasmusvirtual>.

e-teaching.org (2015). Drehbuch, in e-teaching, Projektleitung U. Cress. Tübingen: Leibniz-Institut für Wissensmedien. Zugriff am 27. 3. 2020. URL: $<$ https://www.eteaching.org/didaktik/konzeption/inhalte/drehbuch/index_ht $\mathrm{ml}>$.

Gesetz zum Schutz der Teilnehmer am Fernunterricht (1977). Bonn: Bundesministerium der Justiz und für Verbraucherschutz. Zugriff am 9. 10. 2018. URL: 〈https://www.gesetze-im-internet.de/fernusg/FernUSG.pdf〉.

Grünewald, A. (2010). E-Learning, in Metzler Lexikon Fremdsprachendidaktik, hrsg. v. C. Surkamp (Stuttgart: Metzler): 41-45. 
Guglielmi, L. und Blatešić, A. (2011). Apprendere le microlingue in Teletandem, tra teoria e pratica. Metodički vidici 2: 83-96.

Hagenow-Caprez, M. (2013). Unterrichten mit Szenarien: Aus der Praxis. Babylonia 1: 47-50.

Herzig, B. (2014). Wie wirksam sind digitale Medien im Unterricht? Gütersloh: Bertelmann Verlag.

Hölscher, P., Roche, J., Simic, M. (2009). Szenariendidaktik als Lernraum für interkulturelle Kompetenzen im erst-, zweit- und fremdsprachigen Unterricht. Zeitschrift für Interkulturellen Fremdsprachenunterricht Didaktik und Methodik im Bereich Deutsch als Fremdsprache 14/2: 43-54.

Hradil, S. (2012). Was sind und wozu dienen Szenarien? Zugriff am 23. 3. 2020. URL: <https://www.bpb.de/politik/grundfragen/deutsche-verhaeltnisse-einesozialkunde/139122/szenarien>.

Juchem-Grundmann, C. (2010). Fernunterricht, in Metzler Lexikon Fremdsprachendidaktik, hrsg. v. C. Surkamp (Stuttgart: Metzler): 59-60.

Küppers, A. und Schmidt, T. (2010). Inszenierung, in Metzler Lexikon Fremdsprachendidaktik, hrsg. v. C. Surkamp (Stuttgart: Metzler): 116-117.

Leubner, M., Saupe, A., Richter, M. (2010). Literaturdidaktik. Berlin: Akademie Verlag.

Mehrabian, A. (1971). Silent Messages. Belmont: Wadsworth Publishing Company.

Meister, H. und Shalaby, D. (2014). E-learning: Handbuch für den Fremdsprachenunterricht. München: Hueber Verlag.

Müller-Hartmann, A. (2007). Die Entwicklung interkultureller kommunikativer Kompetenz durch Literatur - Blended Learning in fremdsprachlichen LehrLernkontexten, in Neue Ansätze und Konzepte der Literatur- und Kulturdidaktik, hrsg. v. W. Hallet und A. Nünning (Trier: Wissenschaftlicher Verlag): 197-216.

Nadrljanski, M., Nadrljanski, Đ., Bilić, M. (2009). Digitalni mediji u obrazovanju, in INFuture 2007: Digital Information and Heritage (Zagreb: Faculty of Humanities and Social Sciences): 527-537.

Neustein, A. und López, D. T. (2020). Deutschlernerblog. Zugriff am 3. 4. 2020. URL: <https://deutschlernerblog.de/>.

Pfäffli Tanner, Brigitta K. (2015). Lehren an Hochschulen: Eine Hochschuldidaktik für den Aufbau von Wissen und Kompetenzne. Stuttgart: UTB.

Riechert, R. (1998). Internet im Unterricht Wirtschaftsdeutsch: Erfahrungsbericht aus der Erstellung und Anwendung von Hilfsmitteln für eine effektive Nutzung deutschsprachiger Web-Angebote. Zeitschrift für Interkulturellen Fremdsprachenunterricht [Online] 3/1: 1-10. Zugriff am 23. 03. 2020. 
URL: <http://www.spz.tu-darmstadt.de/projekt_ejournal/jg 03 1/beitrag/ riecher1.htm>.

Rösler, D. und Tschirner. E. (2005). Fremdsprachenlernen mit digitalen Medien: Bemerkungen zum vorläufigen Abschluss einer Diskussion. Deutsch als Fremdsprache: Zeitschrift für Theorie und Praxis des Deutschunterrichts für Ausländer 42/2: 74-79.

Rustemeier-Holtwick, A., Wolpert, A., Sieger, M. (2015). Lernszenario Micro Learning, in Digital lernen - evidenzbasiert pflegen: Neue Medien in der Fortbildung von Pflegefachkräften, hrsg. v. M. Sieger, L. Goertz, A. Wolpert, A. Rustemeier-Holtwick (Berlin: Springer Verlag): 78-84.

Rustemeier-Holtwick, A., Wolpert, A., Lewe, R., Hindenburg, D., Surberg-Finke, G., Bald, U., Buck, G. (2015). Lernszenario Blended Learning, in Digital lernen - evidenzbasiert pflegen: Neue Medien in der Fortbildung von Pflegefachkräften, hrsg. v. M. Sieger, L. Goertz, A. Wolpert, A. RustemeierHoltwick (Berlin: Springer Verlag): 85-108.

Rustemeier-Holtwick, A. und Wolpert, A. (2015). Lernszenario Community of Practice, Digital lernen - evidenzbasiert pflegen: Neue Medien in der Fortbildung von Pflegefachkräften, hrsg. v. M. Sieger, L. Goertz, A. Wolpert, A. Rustemeier-Holtwick (Berlin: Springer Verlag): 109-114.

Schaumburg, H. (2003). Konstruktivistischer Unterricht mit Laptops?: Eine Fallstudie zum Einfluss mobiler Computer auf die Methodik des Unterrichts. (Dissertation). Berlin: Freie Universität.

Son, S. (2004). DaF-Unterricht digital. Deutsch als Fremdsprache: Zeitschrift zur Theorie und Praxis des Deutschunterrichts 41/2: 76-82.

Stoecker, D. (2013). eLearning - Konzept und Drehbuch. Berlin und Heidelberg: Springer-Verlag.

Sullivan, N. und Pratt, E. (1996). A comparative study of two ESL writing environments: A computer-assisted classroom and a traditional oral classroom. System 24: 491-501.

Treml, A. K. und Recker, N. Recker (2004). Lernen, in Einführung in Grundbegriffe und Grundfragen der Erziehungswissenschaft, hrsg. v. H-H. Krüger und W. Helsper (Wiesbaden: Springer Fachmedien Verlag): 103-114.

Tuca, A. (2019). 9 Best Free Blogging Sites: Launch Your Own Blog Without Spending a Dime, in ThemeIsle Blog. Zugriff am 15. 01. 2019. URL: <https://themeisle.com/blog/best-free-blogging-sites/>.

Wannemacher, K., Jungermann, I., Scholz, J., Tercanli, H., Villiez, A. v. (2016). Digitale Lernszenarien im Hochschulbereich. Essen: Verwaltungsgesellschaft für Wissenschaftspflege. 
Wermke, J. (2006). Literatur- und Medienunterricht, in Grundzüge der Literaturdidaktik, hrsg. v. K-M. Bogdal und H. Korte (München: dtv): 91104.

Nikolina N. Zobenica

Univerzitet u Novom Sadu

Filozofski fakultet, Odsek za germanistiku

\section{DIGITALNI MEDIJI I SCENARIJI UČENJA U NASTAVI NEMAČKOG JEZIKA KAO STRANOG}

Rezime

E-učenje ili elektronsko učenje odnedavno je veoma brzo i prilično neočekivano ušlo u sve oblasti obrazovanja, od predškolskog do univerzitetskog. U kratkom roku isprobavaju se i koriste različiti digitalni mediji, bez pripreme i planiranja, i u velikoj meri je zastupljeno učenje putem pokušaja i grešaka. $U$ ovom radu se uz primenu metode teorijske analize: (1) razmatraju različiti pojmovi u vezi sa elektronskim učenjem, kao što su digitalni mediji, učenje na daljinu, hibridna nastava i Blended Learning; (2) iznose se prednosti uvođenja e-učenja (razvijanje digitalnih kompetencija, fleksibilnost mesta i vremena učenja, fleksibilnost sadržaja, mogućnost njihovog ponovnog korišćenja, distribucije, arhiviranja i hipertekstualnog povezivanja, interaktivnost u radu s nastavnim materijalima, kao i u saradnji s nastavnicima i kolegama, uz pomoć alata za komunikaciju i kolaboraciju) i nedostaci (odsustvo paraverbalne i neverbalne komunikacije); (3) navode se mogućnosti implementacije e-učenja u nastavu nemačkog jezika (alati za prezentaciju, vežbanje $\mathrm{i}$ testiranje lingvističkih i jezičkih kompetencija, mogućnosti za upoznavanje s nemačkom kulturom i za razvijanje interkulturnih kompetencija, kreiranje i upotreba blogova, rad s platformama za učenje), uz razmatranje didaktičkih principa (usmerenost na specifične i opšte kompetencije, interakciju, učenike i njihovo aktivno i autonomno učenje, kao i interkulturnu orijentaciju u nastavi); (4) predstavlja se koncept scenarija u opštoj didaktici i didaktici stranih jezika, kao i njegovo povezivanje s novim medijima, s težištem na različitim digitalnim scenarijima učenja prema kriterijumu zastupljenosti digitalnih medija (obogaćivanje, integracija, internet učenje, kolaboracija i interakcija, otvorena obrazovna praksa, igra i simulacija, personalizacija, samostalno učenje) i načina korišćenja medija (tutorski, istraživački, dinamički scenariji učenja). Cilj rada je da se podstakne promišljena i planirana implementacija digitalnih medija u nastavi uopšte, a posebno u nastavi nemačkog jezika kao stranog, kao i da se istakne značaj i neophodnost nastavnika kao organizatora nastavnog procesa u digitalno doba.

Ključne reči: elektronsko učenje, hibridna nastava, didaktički principi, planiranje nastave.

Eingegangen: 29. 4. 2020.

Annahme: 6. 8. 2020. 